\title{
Automatic Detection of Fake Profile Using Machine Learning on Instagram
}

\author{
Er. Pranay Meshram¹, Rutika Bhambulkar², Puja Pokale², Komal Kharbikar², Anushree Awachat ${ }^{2}$ \\ ${ }^{1}$ Assistant Professor, Department of Computer Science and Engineering, Priyadarshini J. L. College of \\ Engineering, Nagpur, Maharashtra, India \\ ${ }^{2}$ BE Scholar, Department of Computer Science and Engineering, Priyadarshini J. L. College of Engineering, \\ Nagpur, Maharashtra, India
}

\section{Article Info \\ Volume 8, Issue 3 \\ Page Number : 117-127}

\section{Publication Issue}

May-June-2021

\section{Article History}

Accepted : 10 May 2021

Published : 14 May 2021

\begin{abstract}
With the arrival of the Internet and social media, at the same time as masses of humans have benefitted from the full-size reassets of records available, there was an full-size boom with inside the upward push of cyber-crimes, mainly targeted closer to women. According to a 2019 file with inside the Economics Times, India has witnessed a $457 \%$ upward push in cybercrime with inside the 5 years span among 2011 and 2016. Most speculate that that is because of effect of social media inclusive of Facebook, Instagram and Twitter on our day by day lives. While those simply assist in growing a legitimate social network, advent of consumer debts in those websites normally desires simply an email-id. A actual lifestyles man or woman can create more than one fake IDs and for this reason impostors can effortlessly be made. Unlike the actual international state of affairs in which more than one policies and guidelines are imposed to become aware of oneself in a completely unique manner (as an instance at the same time as issuing one's passport or driver's license), with inside the digital international of social media, admission does now no longer require this kind of checks. In this paper, we study the one-of-a-kind debts of Instagram, specifically and try and verify an account as fake or actual the use of Machine Learning strategies specifically Logistic Regression and Random Forest Algorithm.
\end{abstract}

Keywords : Fakeprofile, Detection, Machine Learning, Social Media, Instagram, Internet

\section{INTRODUCTION}

In latest years, on line social networks (OSNs), which include Instagram, Twitter, and Facebook, have end up famous structures to disseminate and percentage information [1]. These offerings offer rapid and appropriate conversation and different forms of gear that make their customers be capable of without delay percentage and put up their multimedia contents which include pictures, movies, and audios over the

Copyright: (C) the author(s), publisher and licensee Technoscience Academy. This is an open-access article distributed under the terms of the Creative Commons Attribution Non-Commercial License, which permits unrestricted non-commercial use, distribution, and reproduction in any medium, provided the original work is properly cited 
internet [2]. Hence, except the huge range of customers on social structures, those capabilities and gear have involved many cyber criminals in the use of them to perform their malicious sports on social media structures efficiently. Unlike with inside the beyond, many assaults with a confined or small impact can now have a substantial effect via way of means of the use of on line social structures [3]. However, social medias impact is huge in peoples lives, and plenty of human beings use them to construct extra extensive connections [4]. One of the maximum famous social media is Instagram [5]. Instagram is a loose social networking app built for sharing images and movies over the internet. It is comparable to maximum different social media, wherein folks who create an account have a profile and information feed and may percentage images and movies via that.

In latest years, many celebrities and groups have created their debts on Instagram; they use Instagram to develop their commercial enterprise and fans [6]. Furthermore, a lot of them and different wellknown customers use it as a platform for marketing and marketing. When a person is boosting the range of fans over a hundred thousand or millions, it's far no wonder to apply that humans account as a profitable earner. In the final years, many celebrities and everyday folks who reached a substantial range of fans on Instagram have used their debts as an area for marketing and marketing. People additionally attempt to growth the range in their fans for different reasons, which include attaining extra fame, being honest, and being influential. Such versatility and unfold of use have made Instagram the best platform for the proliferation of ordinary debts, which behave in uncommon ways. Most instructional researchers have basically targeted on spammers and debts, which put their efforts into spreading marketing and marketing, junk mail, malware, and different suspicious sports [7].
These malicious debts are generally the use of computerized applications to enhance their performance, conceal their actual identity, and appear to be actual customers. In beyond years, media have stated that account of celebrities, politicians, and a few famous commercial enterprise has indicated suspicious inflation of fans. Fake Instagram debts particularly used to growth the range of fans of a goal account. Therefore, artificially inflating the range of fans can additionally be concluded to acquire an account extra influential and honest with a view to stand from the gang to gain and entire extra valid fans to their account [8]. In beyond years, a number of the banks and monetary establishments with inside the U.S. determined to investigate social media debts of the mortgage applicants, earlier than sincerely giving the mortgage.

Hence, having a famous account can assist efficiently to growth the creditworthinessand reliability of the applicant. Furthermore, if a spammer followed fake fans, it could efficiently act as a valid person and put up extra authoritative messages and release diverse green marketing and marketing campaigns [9]. Some expert customers assume fake account detection is an smooth undertaking with their policies primarily based totally on anomaly account behaviour. Though, such policies are generally matched neither with analytic algorithms to combination them nor with validation mechanisms. Most instructional researchers have targeted in particular on junk mail and bot detection in diverse social media like Facebook and Twitter, with incredible outcomes in classifying fake debts primarily based totally on their valid and fraudulent features, mainly making use of machine-masteringstrategies.

However, the papers last element proceeds as follows: the second one segment will deliver a short evaluate of the preceding studies in fake and spam debts detection in extraordinary environments and highlights their shortcomings and achievements. The 1/3 segment 
describes the technique used for this study, along with the function extraction, and dataset. The fourth segment presents statistics approximately the proposed detection model, along with the used strategies and detection process. The 5th segment presents experiments along with an outline of the test setup, conditions, and assessment metrics used with inside the test. It additionally discusses the outcomes and the studies findings, besides evaluating the outcomes with different techniques. Finally, the sixth segment sums up the paper with concluding remarks.

\section{RELATED WORK}

Today, social media is growing amazingly fast; those offerings are important for lots human beings in society, particularly for advertising campaigns and celebrities and politicians who try and sell themselves the use of fans and fanatics on social media. Hence, fake money owed created on behalf of human beings and companies may be dangerous and harm to those human beings and corporations reputations and ultimately caused the reducing variety in their actual likes and fans. Moreover, all sorts of fake profiles have an unfavorable impact at the blessings of social media for advertising and corporations in advertising. These fake profiles may be a manner for the cyber bullying; actual customers additionally have extraordinary anxieties approximately their privateness with inside the on-line surroundings with those fake profiles .

Therefore, over the last years, many researchers have investigated the hassle of detecting malicious sports and spammers in social media the use of gadget gaining knowledge of strategies. However, there are a confined variety of studies articles referring to detecting fake money owed or fake fans. In this section, we shed mild on each spammers and fake money owed answers that lately had been added. Ferrara et al. added a technique to stumble on bot customers on Twitter primarily based totally at the fantastically shared capabilities that distinguish them from valid customers. In their proposed approach, they have used a gadget gaining knowledge of approach and behavioral styles among valid and bot money owed so as to classify money owed into the bot or valid class. Cresci et al. have created and used a baseline dataset of verified human and fake fans on Twitter. In their work, they exploited the baseline dataset to educate a fixed of gadget gaining knowledge of classifiers constructed primarily based totally on reviewed policies and capabilities set the use of the media. Their proposed approach is green in detecting fake money owed; the consequences accomplished via way of means of their approach display it can classify greater than $95 \%$ of the money owed successfully from the authentic schooling set.

In a barely extraordinary approach, Zhang and Lu. Introduced a unique approach for the detection of fake money owed in Weibo. Their proposed answer has extraordinary aspects. At first, that they'd this premise why such money owed exist with inside the first place. In the second, they investigated the overlap among fans listing of the clients of fake fans, and that they located a excessive overlap among their follower lists. Their research located 395 nearduplicates, which caused 11.90 million fake money owed that despatched 1,000,000 hyperlinks with inside the network. Thomas et al. made a group of 1.eight million tweets despatched via way of means of 32.nine Twitter money owed. In their research, they located Twitter suspended approximately 1.1 million of these money owed. They have decided on randomly a hundred of these money owed to research their tweets and confirm they had been spamming money owed.

They made a similarly evaluation on that a hundred decided on money owed, and that they locate 93 of the chosen money owed had been suspended for posting junk mail and the unsolicited commercial of numerous products. Three different money owed had been suspended for re-tweeting content material of 
extraordinary information money owed, and the alternative four remained money owed had been suspended for reproduction and competitive advertising posts.

Gao et al. have used a fixed of capabilities for efficiently reconstructing junk mail tweets into campaigns in place of studying them separately. The end result suggests their proposed answer received . However, the disadvantage in their approach is its low detection accuracy. Benevenuto et al. proposed a option to stumble on spammers from non spammers. In their approach, they used an SVM classifier, that is a supervised gadget gaining knowledge of algorithm. They have used 23 conduct and 39 content material capabilities to distinguish spammers from nonspammers, and that they accomplished experiments via way of means of 5 -fold cross-validations. The experiments display they had been nearly a success in figuring out spammers from non-spammers. Bala Anand et al. advanced a new gadget to stumble on fake customers at the Twitter platform the use of a graph-primarily based totally semi-supervised gaining knowledge of algorithm (EGSLA) and examine and amassing behavioral and person-generated content material (UGC) information.

The version first gathered customers information, analyzed them to extract beneficial capabilities, and then accomplished type on those capabilities and made decisions. The experimental consequences display that the EGSLA algorithm accomplished excessive overall performance and turned into greater useful than different algorithms inclusive of choice tree, KNN, SVM, and game theory-primarily based totally techniques in phrases of type accuracy. Sahoo et al. provided a hybrid version to stumble on malicious profiles on social media specializing in Twitter. The proposed

hybrid version consists of modules; first, they analyzed and extracted capabilities the use of Petri internet structure, then they used those capabilities because the classifiers enter to categorize profiles as malicious and legitimates classes. The experimental outcomes display that the proposed method effectively distinguished extraordinary twitter money owed and received a excessive detection fee in phrases of type accuracy.

Therefore, in step with literature, many researchers have been the use of gadget gaining knowledge of strategies to conquer security issues in social networks.Surveyed research typically focused on junk mail detection on microblogging social media. They have investigated many answers to resolve the hassle of junk mail and Fake money owed on Twitter and different microblogging social media. However, to date, there's no complete answer to fake money owed at the Instagram platform, that is one in every of the motivations at the back of this study. Hence, on this paper, we have proposed an green approach for detecting fake money owed on the Instagram platform, that could efficaciously classify extraordinary Instagram person money owed.

\section{METHODOLOGY}

Like maximum social media platforms, the general public Instagram developer API most effective offers the general public facts of customers. It isn't feasible to get right of entry to a few customers sports and login facts, more often than not while a consumer already has set that account to personal mode. This hassle is taken into consideration as an impediment to the system of records series. To remedy the problems and crawl customers facts, we've advanced a selected records crawler and function series device defined with inside the following steps (see Figure 1). 


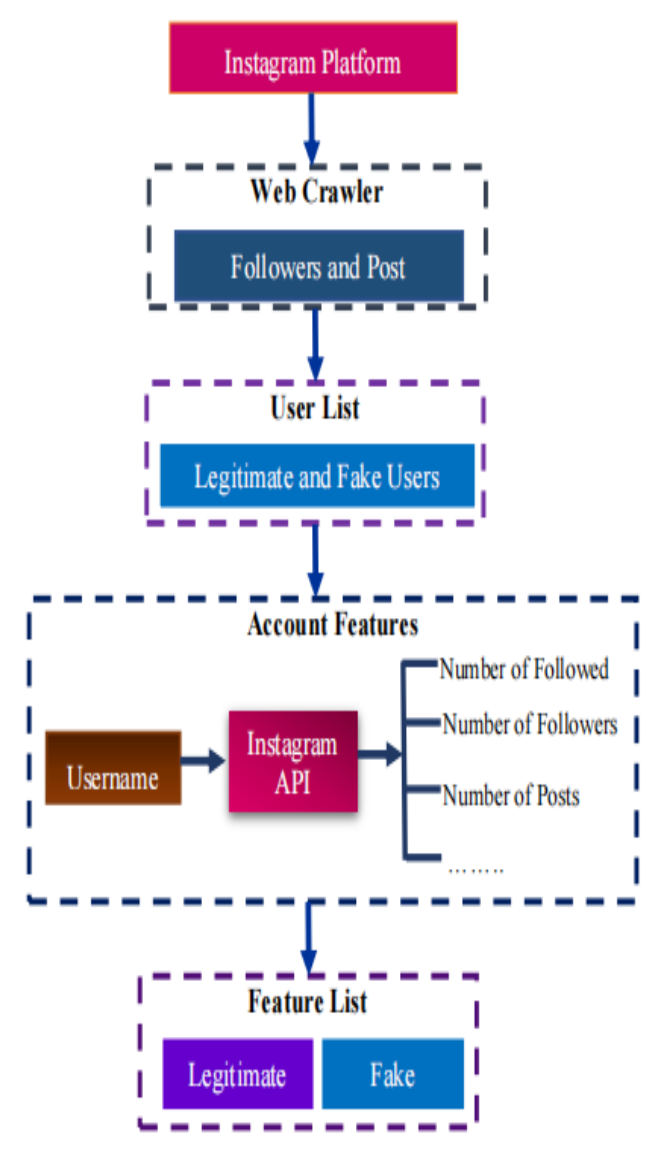

Figure 1. Dataset and feature collection procedure

The 6868 ordinary customers, consisting of celebrities, companies, and everyday valid customers, plus 3132 anomaly customers who manually checked and selected, have gathered with inside the dataset. We have advanced styles of records crawlers for achieving ordinary and anomaly customers, respectively. The everyday user crawler has used Instagrams discover function to discover ordinary customers and upload them to the listing of everyday customers with inside the dataset. Instagrams Explore function shows current published pictures and motion pictures that reached different customers attention, which show debts published are in most cases actual and valid. Furthermore, to find out and reap fake customers on Instagram, first, the advanced crawler used to gain fake customers ID via the follower listing of customers who taken into consideration a widespread variety of fake customers of their follower listing. In second, we've got advanced some other device to manually test all of the fake archived customers with inside the dataset, be confident approximately their identity, and enhance the datasets quality.

\begin{tabular}{cccc}
\hline & Legitimate & Fake & Total \\
\hline Records & 6868 & 3231 & 10000 \\
Percentage & 68.68 & 32.31 & 100 \\
\hline
\end{tabular}

Table 1. Description of the dataset

For every user, a few public records crawled using Instagram API; the outline of the dataset and listing of the crawled capabilities are indexed in Tables 1 and 2 , respectively. The gathered capabilities are indexed with inside the following Table 2.

\begin{tabular}{|c|c|c|}
\hline Index & Feature & Description \\
\hline 1 & UName & Username Length \\
\hline 2 & Uid & Real ID of user on Instagram \\
\hline 3 & Fullname & Full name Length \\
\hline 4 & has_pic & $\begin{array}{l}\text { Does account set a profile } \\
\text { picture }\end{array}$ \\
\hline 5 & biography & Biography Length \\
\hline 6 & Followedby & $\begin{array}{l}\text { The number of users Followed } \\
\text { the account }\end{array}$ \\
\hline 7 & Followed & $\begin{array}{l}\text { The number of users the account } \\
\text { Followed them }\end{array}$ \\
\hline 8 & Is_Followed_More & $\begin{array}{c}\text { Is number of Followed are more } \\
\text { than Followed by }\end{array}$ \\
\hline 9 & Postcount & $\begin{array}{l}\text { The number of shared posts by } \\
\text { the account }\end{array}$ \\
\hline 10 & is_business & Is it a business account \\
\hline 11 & is_private & Is the user set profile as private \\
\hline 12 & is_verified & $\begin{array}{l}\text { Is the account verified by } \\
\text { Instagram }\end{array}$ \\
\hline 13 & has_channel & Does the account have a channel \\
\hline 14 & external_url & $\begin{array}{l}\text { Is the account linked to an } \\
\text { external URL }\end{array}$ \\
\hline 15 & highlight_reel_count & $\begin{array}{l}\text { The number of highlights is } \\
\text { pinned to the account }\end{array}$ \\
\hline 16 & connected_fb_page & $\begin{array}{l}\text { Is the account linked to a } \\
\text { Facebook profile }\end{array}$ \\
\hline
\end{tabular}

Table 2. The list of collected features

Figure 2 illustrated four of the important capabilities in our dataset. Figure 2 (a) indicates the distribution of debts with a profile photograph with inside the dataset, and it suggests maximum debts which have now no longer set profile pix to their debts belong to 
the Fake category. Similarly, the range of observed through fake debts are pretty big, and maximum fake debts observed extra humans than their fans, as proven in Figure 2 (b) and (c). It can be defined that maximum fake customers created best to boom the range of fans of ordinary customers and that is due to the fact many Instagram ordinary customers attempt to shop for repute the usage of boom the range in their debts fans.

Figure 2 (d) suggests the range of posts in every account. As expected, maximum fake debts do now no longer include many posts or in general have 0 or one submit due to the fact a lot of those debts best goal at following different customers or advertising, so their author consumes a brief time at the growing range of their submit or layout look of them. However, the recent author of those fake debts attempts to layout their look and boom the range in their posts, so their debts appearance extra regular and fulfill their customers. Furthermore, through this trick, they are able to now and again bypass Instagrams limitations. Though, their account nonetheless has a small range of fans and a big range of following. Some of those fake account manufacturers attempted to lower those vast variations among the numbers in their debts fans and observed through the usage of the alternative fake debts they own.

Accordingly, they make fake sports among their fake debts and make their fake debts that are in general bots following every different to their range of fans appearance like ordinary customers. Finally, all in their fake debts could have some fans, and it facilitates them appearance extra like ordinary customers.

\section{PROPOSED WORK}

This segment is associated with the detection of Fake debts.Fake debts are the debts which can be used to increase the recognition metrics of different users. For this reason, they have a propensity to have a excessive following and occasional follower counts. Their liking conduct can also additionally appearance randomly. The absence of profile photograph and odd person names are the common traits of Fake debts. In this segment, the dataset and the chosen capabilities for the dataset had been brought for the detection of Fake account. Then, the oversampling approach is defined which is essential for the unevenness with inside the variety of actual and Fake account with inside the dataset.

\section{A. Dataset and Features}

For the dataset, 1002 actual account and 201 Fake account has been collected after considerable guide labeling, including debts from one-of-a-kind international locations and fields. During this gathering process, the factors this is paid interest are follower and following counts, media counts, media posting dates or frequency, remarks on media, a number of the accompanied and

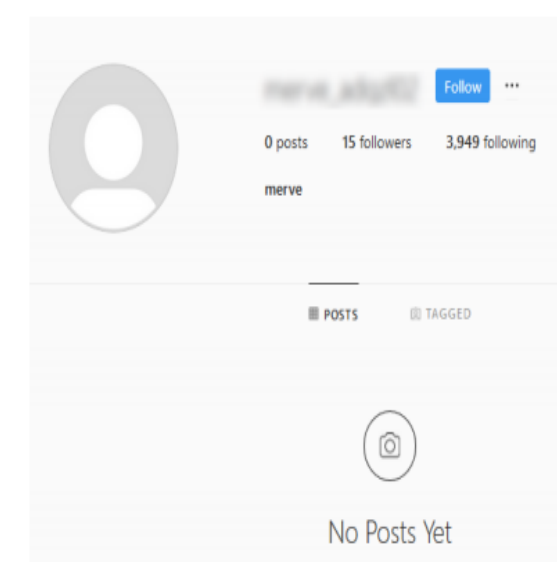

Figure 2: Fake account example from dataset.

Most suspicious accounts are labeled as fake by hand. 


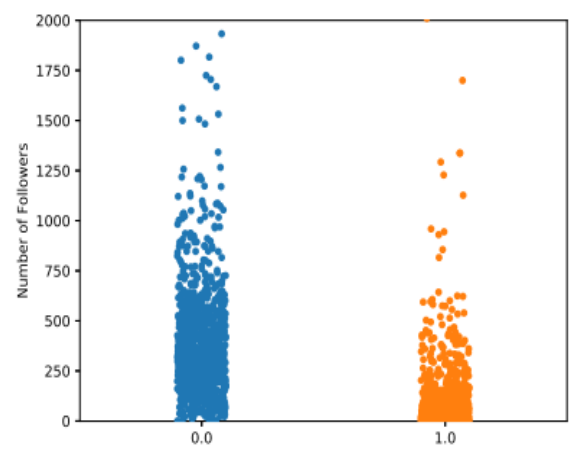

Figure 3: In-class data distributions for "follower count" feature.

\footnotetext{
1. user_media_count - Total number of posts, an account has.

2. user_follower_count - Total number of followers, an account has.

3. user_following_count - Total number of followings, an account has.

4. user_has_profil_pic - Whether an account has a profil picture, or not.

5. user_is_private - Whether an account is a private profile, or not.

6. user_biography_length - Number of characters present in account biography.

7. username_length - Number of characters present in account username.

8. username_digit_count - Number of digits present in account username.

9. is_fake - True, if account is a spam/fake account, False otherwise
}

Table 6 : Dataset Features and Snapshot of training data

following account, the life of profile photo and the username of the profile.

An instance Fake profile from the dataset may be visible. As visible, it has a excessive following variety of 3949, and coffee follower variety of 15 , has no profile photo and no published media.

In the dataset, the chosen base functions may be indexed as below:

- Total media variety of the account.

- Follower remember of the account.

- Following remember of the account.

- Number of digits found in account username.

Whether account is private, or not (binary feature). To emphasize, all of the functions aren't associated with the user media, consequently the set of rules isn't suffering from the account privacy. The motive to feature variety of digits found in account username is that in the era of Fake money owed, some money owed are produced through including distinctive numbers to the same name. The variety of digits distribution may be visible .

As visible, greater that $50 \%$ of Fake money owed have greater than one digits at the same time as actual account has no digits with approximately $89 \%$.

\section{B. Oversampling}

Distribution of training with inside the Fake account dataset isn't even. This effects in negative overall performance for the outnumbered class. SMOTE oversampling technique is applied to growth variety of samples for Fake money owed. $\mathrm{K}$ is selected as 5 for this work. In the implementation of SMOTE, SMOTE-NC is applied which considers not only the quantity classes but also the the categorical classes. After applying oversampling, all classifiers are trained on equal number of training samples per class (1002 per class).

\begin{tabular}{ccc}
\hline \# of digits & Real accounts & Fake account \\
\hline $\mathbf{0}$ & $88.9 \%$ & $46.8 \%$ \\
$\mathbf{1}$ & $2.5 \%$ & $10.0 \%$ \\
$\mathbf{2}$ & $5.3 \%$ & $13.9 \%$ \\
$\mathbf{3}$ & $0.7 \%$ & $11.4 \%$ \\
$\mathbf{3 +}$ & $2.6 \%$ & $17.9 \%$ \\
\hline
\end{tabular}

TABLE 7: Distribution of accounts with changing number of digits included in their usernames

\section{ANN}

Artificial Neural networks (ANN) or neural networks are computational algorithms.It intended to simulate the behavior of biological systems composed of "neurons". ANNs are computational models inspired by an animal's central nervous systems. It is 
capable of machine learning as well as pattern recognition. These presented as systems of interconnected "neurons" which can compute values from inputs.

A neural network is an oriented graph. It consists of nodes which in the biological analogy represent neurons, connected by arcs. It corresponds to dendrites and synapses. Each arc associated with a weight while at each node. Apply the values received as input by the node and define Activation function along the incoming arcs, adjusted by the weights of the arcs.

A neural network is a machine learning algorithm based on the model of a human neuron. The human brain consists of millions of neurons. It sends and process signals in the form of electrical and chemical signals. These neurons are connected with a special structure known as synapses. Synapses allow neurons to pass signals. From large numbers of simulated neurons neural networks forms.

\section{Random forest Algorithm}

This is a regression version appearing properly on category version. Since there are a totally few assumptions connected to it, so information guidance is much less challenging. We used the random Forest feature in $\mathrm{R}$ and set the $\mathrm{n}$-tree parameter (denoting the quantity of bushes) as 500 and the quantity of variables attempted at every cut up as 3 . Random Record Selection is the primary challenge wherein every tree is educated on $2 / 3$ of the total education information and a few variables are decided on at random(say $\mathrm{m}$ ) out of all of the variables and those $\mathrm{m}$ variables are used to cut up the node. For every tree, the use of the leftover (36.8\%) information, the misclassification charge is calculated. This offers us the Out Of Bag (OOB) mistakes charge. The woodland chooses the category having the maximum votes over all of the bushes with inside the woodland. This is the
$\mathrm{RF}$ rating and the percentage YES votes acquired is the expected probability.

\section{SVC}

SVC is one of the most popular Supervised Learning algorithms, which is used for Classification as well as Regression problems. However, primarily, it is used for Classification problems in Machine Learning.

The goal of the SVC algorithm is to create the best line or decision boundary that can segregate ndimensional space into classes so that we can easily put the new data point in the correct category in the future. This best decision boundary is called a hyperplane.

SVC chooses the extreme points/vectors that help in creating the hyperplane. These extreme cases are called as support vectors, and hence algorithm is termed as Support Vector Machine. Consider the below diagram in which there are two different categories that are classified using a decision boundary or hyperplane.

In this section we will discuss about the clusters we formed and in the table we have key value pairs which will have the group of pairs. This will tell the medication limit of the patient based on the age, gender, weight, previous treatments, previous medication, and current scenario. The below is the table which will give the explanation based in the key value pair. This pair will give the warning to the doctor if her give the high drug usage. These are the few clustering results we acquired as mentioned below which will have the values of how many times a pair of key value is being repeated.

In this section we determine the results of the two classification models.After creating the models using the training datasets, we apply the models on unseen data, i.e., and the test dataset. We create the 
Er. Pranay Meshram et al Int J Sci Res Sci \& Technol. May-June-2021, 8 (3) : 117-127

confusion matrix based on these and calculate various performance parameters as discussed below.

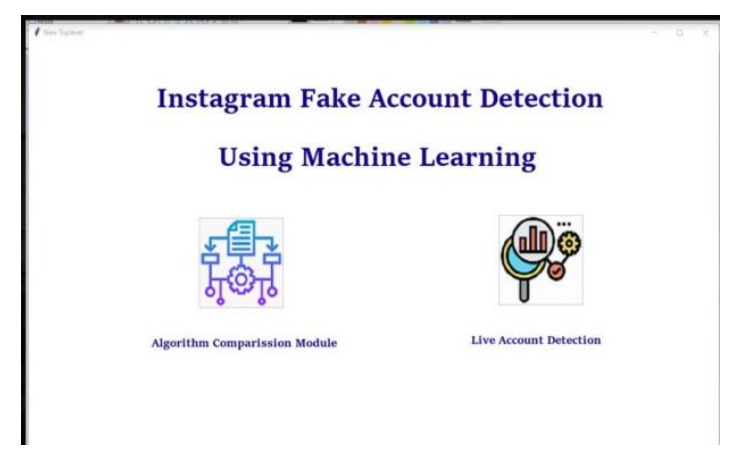

Figure 4 : Home Screen Module

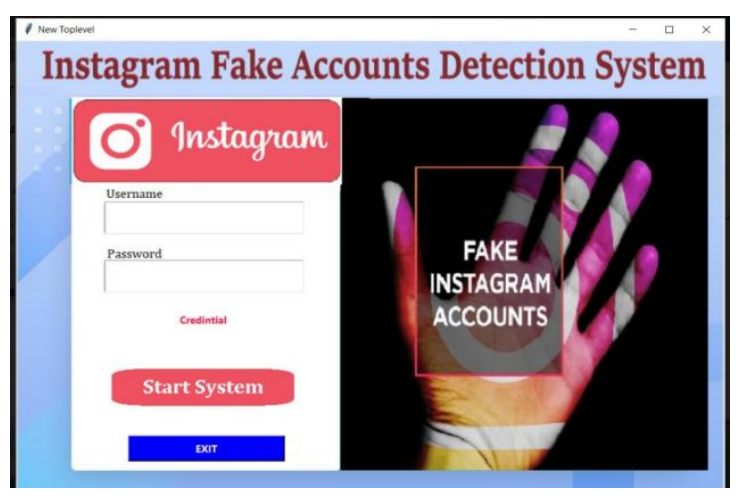

Figure 5: LoginModule

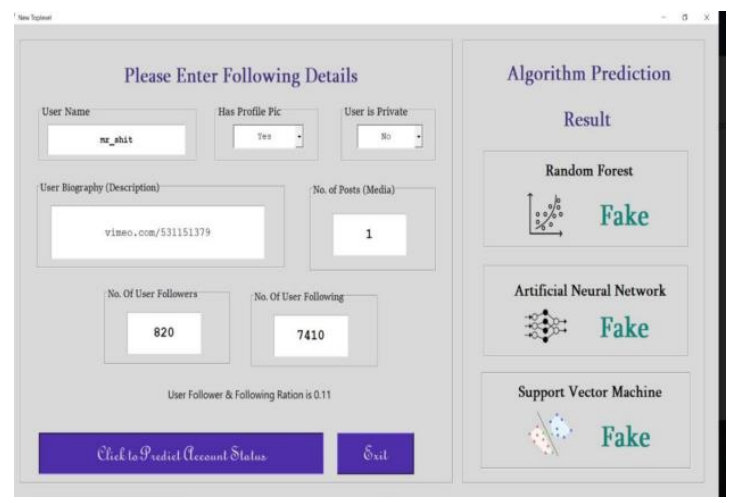

Figure 6: Live prediction Module

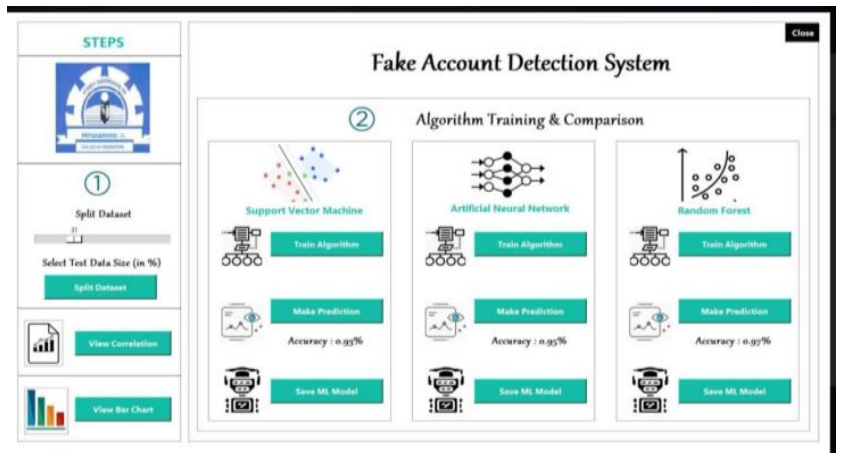

Figure 7 : Algorithm Accuracy Comparison Module

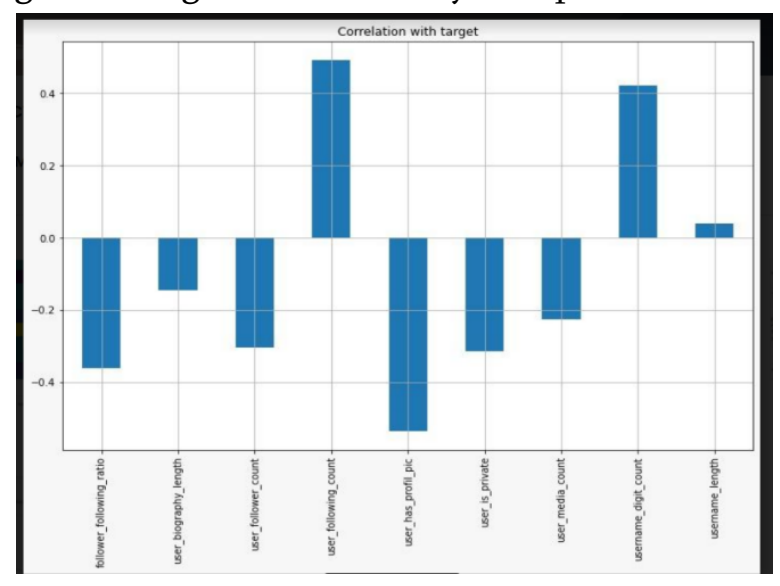

Figure 8 : Graph based on dataset
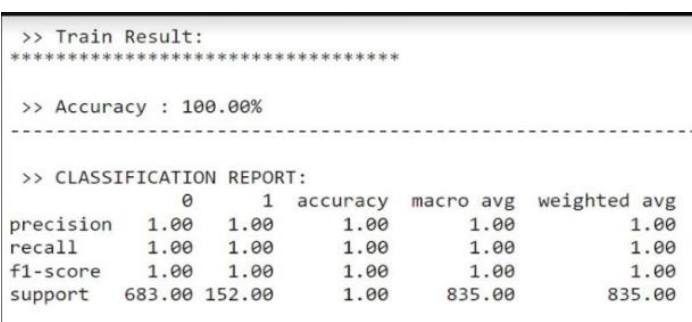

>> Testing Result:

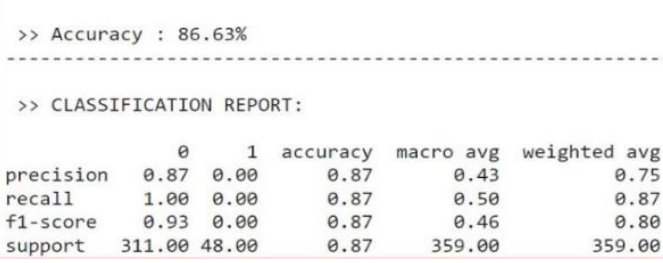

Figure 10 : Accuracy of SVC Algorithm 


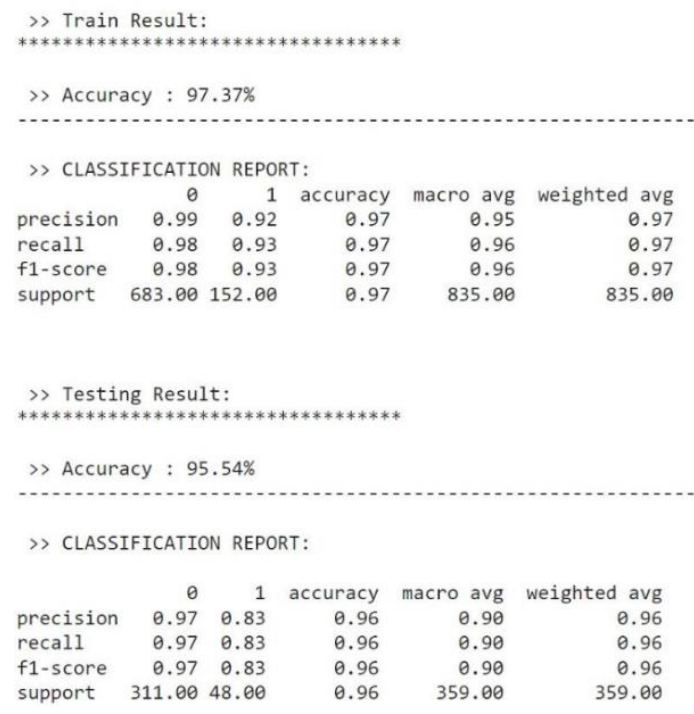

Figure 11 : Accuracy of ANN Algorithm

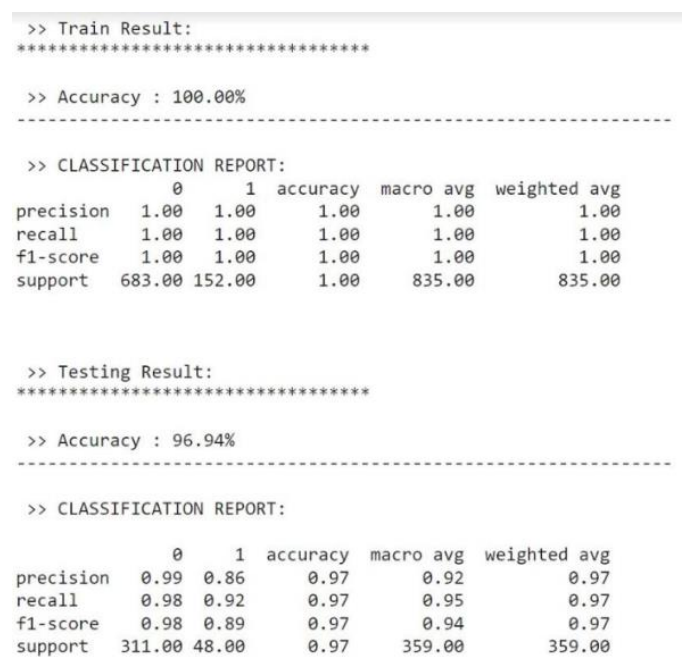

Figure 12 : Accuracy of Random Forest Algorithm

\section{v. CONCLUSION}

The final results of this studies is essential for commercial enterprise proprietors who sit up for finding influencers for logo endorsement. Unlike Twitter, that is utilized by maximum studies, Instagram has richer functions in media sharing, and it's far confirmed to be the maximum used platform for logo marketing. Possible development for this studies is the inclusion of textual content evaluation in each caption and remarks, relation graph evaluation, and photograph evaluation. Some captions of the Fake customers are irrelevant, such as "observe me", and some remarks given to them also are coming from Fake customers. By doing this, the Fake customers can keep away from being banned via way of means of Instagram via way of means of preserving properly engagement levels. Image evaluation is likewise beneficial due to the fact that many spammers submit textual content-primarily based totally images. Graph evaluation is likewise useful for the type due to the fact that a number of the Fake customers are with inside the identical circle of relation. Therefore, thru a multitude of analysis, experiment, evaluation, and implementation work, the experiments consequences have shown that the proposed technique is viable and able to classifying over $98 \%$ of customers accurately.

\section{FUTURE WORK}

Detection of the Fake and automatic money owed which results in Fake engagement in Instagram is studied as a binary classification trouble on this paper. To our knowledge, that is the fit first time for such an evaluation over Instagram money owed. Our contributions with this paintings are: series of datasets for Fake and automatic account detection, proposing derived functions for Fake and automatic classification, proposing a fee touchy function discount method primarily based totally on genetic algorithms for choosing high-quality functions for the classification of computerized money owed, correcting the unevenness with inside the Fake account dataset the usage of the algorithm and comparing numerous sample reputation strategies over the accrued datasets.

\section{REFERENCES}

[1]. Rohit Raturi Associate Director, Enterprise Solutions,KPMG, USA LP, Montvale, NJ, USA. "Machine Learning Implementation to Identify the Fake Account in Social Networking Sites". International Journal of Pure and Applied 
Mathematics Volume 118 No. 20 2018, 47854797 ISSN: 1314-3395 .

[2]. Ciresan, Dan, Ueli Meier, and Jurgen Schmidhuber. "Multi-column Deep Learning Neural Networks forImage Classi_cation.". IEEE Conference on Computer Vision and Pattern Recognition. (2012): 3642-649.IEEE. Web.

[3]. Hsu, Chih-Wei, and Chih-Jen Lin. "A Comparison of Methods for Multiclass Support Vector Machines.".IEEE Transactions on Neural Networks. 13.2 (2002): 415-25. Web.

[4]. Knerr, S., L. Personnaz, and G. Dreyfus."Singlelayer Learning Revisited: A Stepwise Procedure for Building and Training a Neural Network."Springer. 68 (1990): 41-50. Web.

[5]. Liu, Cheng-Lin, Kazuki Nakashima, Hiroshi Sako, and Hiromichi Fujisawa. "Handwritten Digit Recogni-tion: Investigation of Normalization and Feature Extraction Techniques.". Pattern Recognition. 37.2 (2004):265-79. Web.

[6]. S. Rodriguez, "Instagram Could Delete Up To 10 Million Accounts As It Cracks Down On Spam," International Business Times, 12 November 2014. Online].Available:https://www.ibtimes.com/insta gram-could-delete-10- million-accounts-itcracks-down-spam-1749914. Accessed 4 September 2019].

[7]. E. G. Ellis, "Fighting Instagram's \$1.3 Billion Problem-Fake Followers," Wired, 9 October $2019 . \quad$ Online]. Available:https://www.wired.com/story/instagra m-fake-followers/. Accessed 14 September 2019].

[8]. J. Neff, "Study of Influencer Spenders Finds Big Names, Lots of Fake Followers," 23 April 2018. Online].

Available:https://adage.com/article/digital/studyinfluencer-spenders-finds-big-names-fakefollowers/313223. Accessed 14 September 2019].

[9]. P. G. Efthimion, S. Payne and N. Proferes, "Supervised Machine Learning Bot Detection
Techniques to Identify Social Twitter Bots," SMU Data Science Review,vol. 1, no. 2, 2018.

\section{Cite this article as :}

Er. Pranay Meshram, Rutika Bhambulkar, Puja Pokale, Komal Kharbikar, Anushree Awachat, "Automatic Detection of Fake Profile Using Machine Learning on Instagram", International Journal of Scientific Research in Science and Technology (IJSRST), Online ISSN : 2395-602X, Print ISSN : 2395-6011, Volume 8 Issue 3, pp. 117-127, May-June 2021. Available at doi : https://doi.org/10.32628/IJSRST218330 Journal URL : https://ijsrst.com/IJSRST218330 